\title{
Drei neue Polychaeten-Arten der Familien Polynoidae und Syllidae von Neu-Kaledonien, assoziiert mit einer verkalkten Hydrozoe
}

\author{
Gesa Hartmann-Schröder* \\ Biologische Anstalt Helgoland; Notkestr. 31, D-W-2000 Hamburg 52, Bundesrepublik \\ Deutschland \\ Zoologisches Institut und Zoologisches Museum der Universität; Martin-Luther-King- \\ Platz 3, D-W-2000 Hamburg 13, Bundesrepublik Deutschland *
}

\begin{abstract}
Three new species of polychaetes of the families Polynoidae and Syllidae from New Caledonia, associated with a calcified hydrozoan. Three new species are described: Lagisca zibrowii (Polynoidae) and Procerastea hydrozoicola and P. parasimpliseta (Syllidae), living in association with a calcified hydrozoan, probably of the genus Pseudosolanderia (Rosalindidae). The genus Paraprocerastea is synonymized with Procerastea.
\end{abstract}

\section{EINLEITUNG}

Die hier beschriebenen Polychaeten stammen von drei verschiedenen französischen Forschungsreisen: Chalcal 2 (1986), Musorstom 6 (1989) und SmiB 5 (1989). Diese Fahrten führten in die weitere Umgebung von Neu-Kaledonien im Südwestpazifik, letztere zwei organisiert von B. Richer de Forges und A. Crosnier (vgl. Richer de Forges, 1990).

Das Material wird im Naturhistorischen Museum in Paris (MNHN = Muséum National d'Histoire Naturelle Paris) oder im Zoologischen Museum der Universität Hamburg (ZMH) aufbewahrt.

Alle drei Polychaeten-Arten wurden von $\mathrm{H}$. Zibrowius von verkalkten Hydrozoen abgesammelt, die alle derselben Art angehören und vermutlich der Gattung Pseudosolanderia Bouillon \& Gravier-Bonnet zuzuordnen sind (Zibrowius, lit.).

Lagisca zibrowii sp. n. lebt auf der Oberfläche der Hydrozoen-Kolonie in einer Furche (Abb. 1), während sich die beiden Procerastea-Arten in weichen, papierartigen Sekretröhren längs einiger Äste der Kolonie fanden, frei oder teilweise vom Kalkskelett der Kolonie überwachsen.

Die Beziehungen zwischen den Polychaeten und der Hydrozoe sind auf Grund dieser Funde allein nicht zu klären. Möglicherweise handelt es sich bei L. zibrowii sp. $\mathrm{n}$.

- Mitglied der Taxonomischen Arbeitsgruppe an der Biologischen Anstalt Helgoland

- Korrespondenzanschrift

(C) Biologische Anstalt Helgoland, Hamburg 


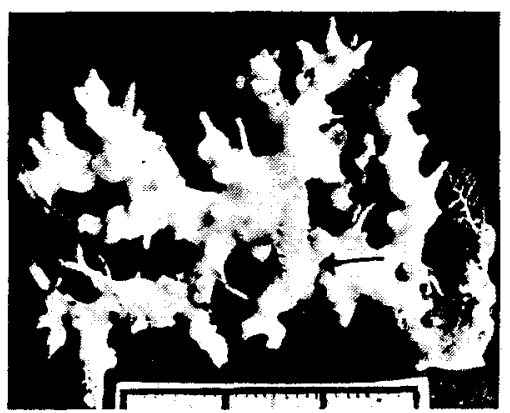

Abb. 1. Hydrozoenkolonie mit Lagisca zibrowii n. sp. in einer Furche (Pfeil); Skala: $3 \mathrm{~cm}$

um Kommensalismus, da es unter den Polynoiden eine ganze Reihe von Arten gibt, die kommensalisch mit verschiedenen Invertebraten leben. Einige nahe Verwandte der Gattung Procerastea aus der Gattung Autolytus leben u. a. auch in Europa in Sekretröhren an Hydrozoen-Kolonien verschiedener Arten und fressen die Polypenköpfchen ab. Demnach handelt es sich wahrscheinlich auch bei den beiden neuen Procerastea-Arten um Räuber. Da sie in ihren Röhren eine mindestens hemisessile Lebensweise haben, könnte man auch von einem Übergang zum Parasitismus sprechen.

\section{BESCHREIBUNG DER ARTEN}

Familie Polynoidae Malmgren, 1867

Unterfamilie Harmothoinae Horst, 1917

Gattung Lagisca Malmgren, 1867

Lagisca zibrowii sp. $\mathrm{n}$.

Die Art widme ich Herrn Dr. Helmut Zibrowius, Station Marine d'Endoume, Marseille, der die Polychaeten von den Hydrozoen absammelte und mir zur Bearbeitung zur Verfügung gestellt hat.

L. zibrowii sp. n. (Abb. 2). Hol o ty p u s mit 49 Segmenten 13-14 mm lang, stark gekrümmt, möglicherweise hinten wenige Segmente fehlend. Einige vordere Segmente mit schwach bräunlichen kurzen Querlinien in der Mitte; außerdem etwas bräunliches Pigment subdistal an den keulenförmig verdickten Cirren. Prostomium mit gerundeten vorderen Ecken. Vier Augen, die vorderen dorsolateral bis lateral auf der Höhe der größten Breite des Prostomiums, die hinteren in den hinteren Ecken (Abb. 2 A). Mediane Antenne mit großer Ceratophore vorn im Einschnitt des Prostomiums, zylindrisch bis keulenförmig, relativ dick, subdistal gerundet und mit distalem fadenförmigen Fortsatz. Laterale Antennen in ventraler Position, ähnlich geformt wie die mediane, jedoch viel kleiner. Antennen erscheinen glatt. Palpen mit winzigen Papillen. 1. Segment mit 0-1 Borste und zwei Paar Tentakelcirren von gleicher Gestalt wie die mediane Antenne (Abb. 2A). 15 Paar Elytren vorhanden, in der Anordnung wie bei Harmothoe, die letzten am 32. Segment, bis hierhin den Rücken vollständig bedeckend, nur Hinterende frei. 
Umriß der Elytren mehr oder weniger nierenförmig, am Vorderrand manchmal leicht gewellt; farblos, dicht und gleichmäßig mit kleinen dornförmigen Mikropapillen bedeckt (Abb. 2B); keine fransenartigen Randpapillen. Dorsaltuberkeln klein, abgerundet dreieckig, unauffällig. Dorsalcirren ähnlich wie die Tentakelcirren (Abb. 2A) und die mediane Antenne; Ventralcirren kleiner, spindelförmig, glatt (Abb. 2C). Notopodien etwas kleiner als Neuropodien, spitz kegelförmig, mit dicker Acicula und etwa elf unterschiedlich dicken und verschieden langen Dorsalborsten, die kleineren vorn und oben im Notopod. Alle Dorsalborsten dicht und fein gezähnt, teilweise mit relativ langen, nackten Spitzen (Abb. 2D). Neuropodien mit schmalem aciculären Lappen, der distal oberhalb der Acicula cirrenförmig verlängert; postsetaler Lappen breit gerundet (Abb. 2C). Etwa 50 Borsten pro Neuropod; sie sind weniger als halb so dünn wie die dicksten Dorsalborsten, mit relativ langer, schlanker, gezähnter Region, subdistal nackt und alle, auch die untersten, mit kurzem sekundären Zahn (Abb. 2E). Eier in der Leibeshöhle.

Locus typicus: ChalCal 2, Stat. DW 76: Banc Stylaster auf der Norfolk-Rippe $\left(23^{\circ} 40,50^{\prime} \mathrm{S}-167^{\circ} 45,20^{\prime} \mathrm{O}: 470 \mathrm{~m}, 30.10 .1986\right.$ : Holotypus (MNHN : UC 227).

Größter Paratyp von Musorstom mit 39 Segmenten (vollständig) etwa $12 \mathrm{~mm}$ lang (gekrümmt). Färbung in Alkohol weißlich, nur auf den mittleren Segmenten

$2 \mathrm{~A}$

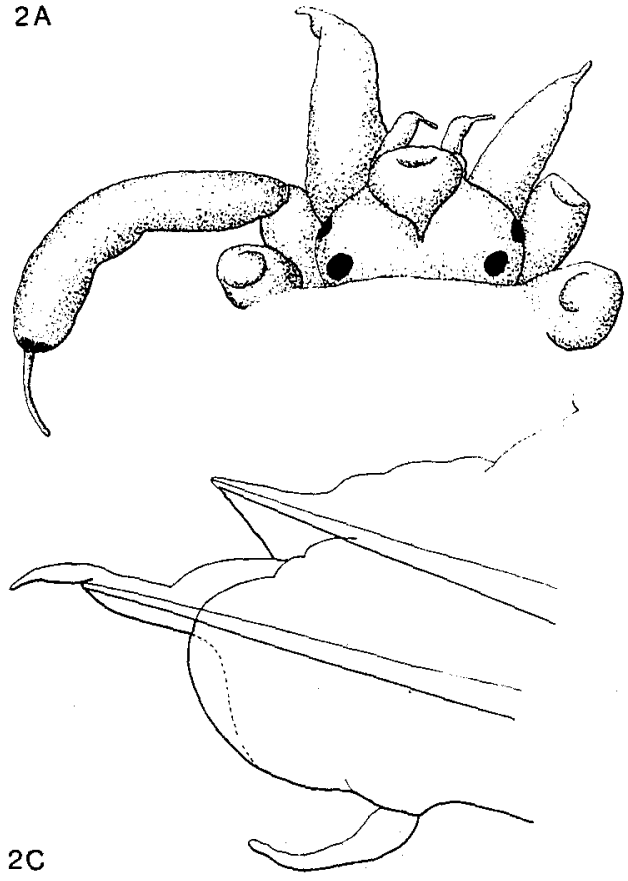

$2 B$

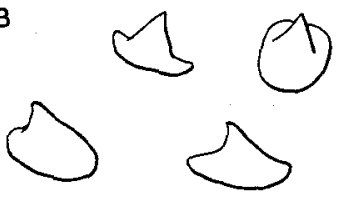

$\left\{\begin{array}{l}y \\ y \\ y\end{array}\right.$

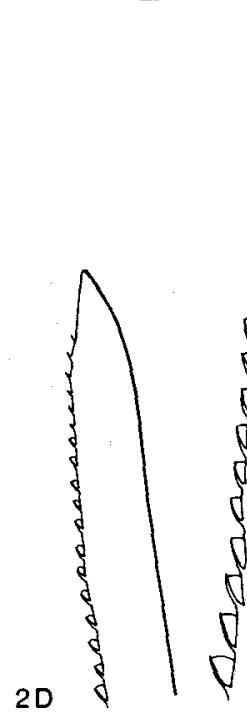

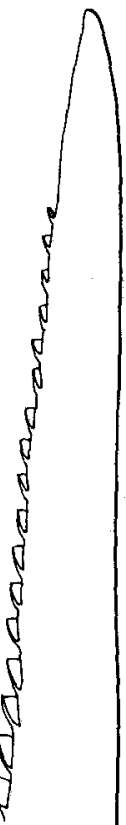

Abb. 2. Lagisca zibrowii n. sp. A: Vorderende eines Paratyps von dorsal (mediane Antenne abgebrochen); B: Elytrenpapillen (verschiedene Blickwinkel); C: mittleres Parapod von hinten; D: Spitzen von Dorsalborsten; E: Spitze einer Ventralborste 
Andeutung eines bräunlichen Pigmentmusters, unterbrochen durch einen hellen Querstreifen und zwei dorsolaterale helle Längslinien. Laterale Antennen eher spindelförmig, etwas kürzer als das Prostomium. Nephridialpapillen an vorderen Segmenten winzig, ab 9. Borstensegment deutlicher, oval, danach länger werdend, fingerförmig. Nach der Verteilung der Elytren eines unvollständigen Paratyps (Hinterende) hätte dieses Tier 48 Segmente gehabt.

Fundort: Musorstom 6, Stat. DW 472: Mont Musorstom auf der Rippe der lles Loyauté $\left(21^{\circ} 08,60^{\prime} \mathrm{S}-167^{\circ} 54,70^{\prime} \mathrm{O}\right): 300 \mathrm{~m}, 22.2$. 1989: 2 Paratypen (1 vollständiges Tier, 1 mittleres Bruchstück und 1 Hinterende) (MNHN:UC 229) und 1 Paratyp (ZMH: P-20 630).

Ein in zwei Teile zerbrochener Paratyp von SMIB besitzt 43 oder 44 Segmente. Etwas braunes Pigment auch an der Basis der kleinen subterminalen Schwellung der Palpen, an der Basis der großen Ceratophore der medianen Antenne und am Hinterrand des Prostomiums. Eier in der Leibeshöhle.

Fundort: SMIB 5, Stat. DW 103: Banc Aztèque auf der Norfolk-Rippe $\left(23^{\circ} 17,4^{\prime} \mathrm{S}-168^{\circ} 04,8^{\prime} \mathrm{O}\right): 315 \mathrm{~m}, 14.9 .1989$ : 1 Paratyp (MNHN : UC 228).

Taxonomische Bemerkung: Auf Grund der Zahl der Elytren (15 Paar) und ihrer Anordnung sowie der Tatsache, daß die hinteren Segmente nackt $-d$. h. nicht von Elytren bedeckt - sind, wird die neue Art nach dem im Augenblick bestehenden System der Gattung Lagisca zugeordnet. Verschiedene Autoren, darunter M. Pettibone und R. Hanley, sind intensiv mit der Systematik der Familie Polynoidae beschäftigt, so daß die Merkmale der Gattung Lagisca stärker eingeschränkt werden könnten und die neue Art möglicherweise später in eine neue Gattung überführt werden muß.

Auffällig bei der neuen Art ist einmal die Form der medianen Antenne, der Tentakelund der Dorsalcirren: keulenförmig, subdistal breit gerundet und distal mit dünnem, fadenförmigen Fortsatz. Diese Form ist bei all den genannten Anhängen und auch bei allen Paratypen vorhanden, so daß sie nicht auf Zufälligkeit im Augenblick der Fixierung beruhen kann. Ein weiteres bedeutendes Merkmal ist das Fehlen von Papillen an Antennen und Cirren. Durch diese Merkmale fällt die neue Art etwas aus dem Rahmen der Gattung Lagisca. Da jedoch Zahl und Anordnung der Elytren sowie Position der lateralen Antennen die Hauptcharakteristika der Polynoiden-Gattungen sind, kann die neue Art im Augenblick nur der Gattung Lagisca zugeordnet werden.

Innerhalb der Gattung Lagisca ist $L$. hubrechti (McIntosh) der neuen Art am ähnlichsten, besonders in der Form der kleinen dorn- oder kegelförmigen Elytrenpapillen und in der Form der Ventralborsten mit langer, gezähnter Region. Bei $L$. hubrechti sind jedoch die Antennen und Dorsalcirren schlank und höchstens mit schwacher subterminaler Schwellung versehen. Außerdem sind die Dorsalborsten nicht so dicht gezählt, und das Prostomium weist kleine vordere Ecken auf, die bei der neuen Art fehlen.

Familie Syllidae Grube, 1850

Unterfamilie Autolytinae Langerhans, 1879

Gattung Procerastea Langerhans, 1884 emend.

Di a g n o s e: Körper ohne segmentale Wimperringe. Prostomium mit vier Augen. Palpen wenig entwickelt, ventralwärts umgeschlagen. Paarige Nuchalwülste (Epaulet- 
ten) oder eine Nuchallamelle am Hinterrand des Prostomiums. Zwei Paar Tentakelcirren. Zylindrische lange Dorsalcirren am 1. Borstensegment; folgende Borstensegmente mit oder ohne Dorsalcirren. Parapodien mit inneren Parapodialdrüsen. Zwei bis drei Analcirren. Zusammengesetzte Borsten mit sehr kurzen, falcigeren Endgliedern; dazu einfache ein- oder zweispitzige Borsten, die aus zusammengesetzten Borsten durch Verschmelzung von Endglied und Schaft entstanden sind; einige Arten nur mit einfachen Borsten. Eine einfache obere Borste distal breit. Pharynx gewöhnlich mit Trepan, bei epitoken Sexualstadien fehlend. Stolone (Sexualstadien) mit blattförmigen oder reduzierten Dorsalcirren, sonst wie bei Autolytus Grube; sechs vordere Segmente ohne Schwimmborsten. Bildung der Stolone einzeln am 14. Borstensegment. Auch vegetative Reproduktion durch Teilung des ungeschlechtlichen (atoken) Tieres und Regeneration der fehlenden Vorder- und Hinterenden.

\section{Procerastea hydrozoicola sp. n.}

P. hydrozoicola sp. n. (Abb. 3). Hol o t y p u s mit 30 Borstensegmenten etwa $5 \mathrm{~mm}$ lang (Tier ist gewunden); größte Breite ohne Parapodien 0,40 mm. Vordere Segmente dorsal sekundär geringelt. Rötlich-braunes Pigment auf dem vorderen Rücken. Prostomium und Palpen nicht deutlich voneinander getrennt, zusammen etwa so lang wie breit. Vier Augen im Trapez angeordnet, die vorderen wenig größer (Abb. 3A). Antennen abgebrochen, die Ceratophore der medianen Antenne zwischen den vorderen Augen, die Ceratophoren der lateralen Antennen weiter vorn, vor den Augen. Mediane Antenne lose neben dem Tier in der Röhre liegend, lang, zylindrisch. Nuchal-Epauletten am Hinterrand des Prostomiums, bis etwa zum Ende des 2. Borstensegments reichend. Dorsale Tentakelcirren zylindrisch, etwas weiter als die Palpenspitzen nach vorn ragend. Ventrale Tentakelcirren etwa halb so lang (Abb. 3A). '1. Dorsalcirren abgebrochen, fehlend, wahrscheinlich lang. An allen folgenden Segmenten auch Dorsalcirren vorhanden; diese kurz, wenig unterschiedlich, finger- bis spindelförmig (Abb. 3A). Keine Ventralcirren. Parapodien kurz, gerundet, besonders im ventralen Bereich polsterartig und drüsig. Vordere Parapodien mit bis zu 20 Borsten, davon 1-3 untere deutlich zusammengesetzt; mittlere Parapodien mit 13-15 Borsten, davon 2 untere zusammengesetzt; hintere Parapodien mit 10 Borsten, davon 2-3 untere zusammengesetzt. Einfache Borsten mit subdistaler Einschnürung, distal bifid, wobei der sekundäre Zahn viel größer als der terminale (Abb. 3D). Zusammengesetzte Borsten mit unregelmäßig gezacktem Schaft distal und kurzem bifiden Endglied mit großem sekundären Zahn (Abb. 3B-D). Dazu ab 15. Parapod eine einfache obere Borste, die subdistal verbreitert und distal mit sechs kurzen und einem langen Randzahn (Abb. 3D). Pharynx anscheinend mit großer Schleife; Trepan nicht genau zu erkennen. Magen von der Mitte des 4. bis Ende des 6. Borstensegments, mit 21 oder 22 Muskelzellreihen.

Pa ratyp: 30 Borstensegmente $=3,5 \mathrm{~mm}$. Tier ohne Darm, dafür mit unreifen Gameten. Es handelt sich demnach um einen möglicherweise durch das Sammeln zu früh abgelösten Stolon. Vordere Dorsalcirren etwas länger als beim atoken Holotypus, jedoch immer kürzer als Segmentbreite. Borsten völlig übereinstimmend mit dem Holotypus. Holotypus und Paratyp in papierartiger Röhre.

L o cus ty pi cu s: Musorstom 6, Stat. 472: Mont Musorstom auf der Rippe der lles Loyauté $\left(21^{\circ} 60^{\prime} \mathrm{S}-167^{\circ} 54,70^{\prime} \mathrm{O}\right): 300 \mathrm{~m}, 22.2$. 1989: Holotypus (MNHN : UC 230) und 1 Paratyp (MNHN : UC 231). 

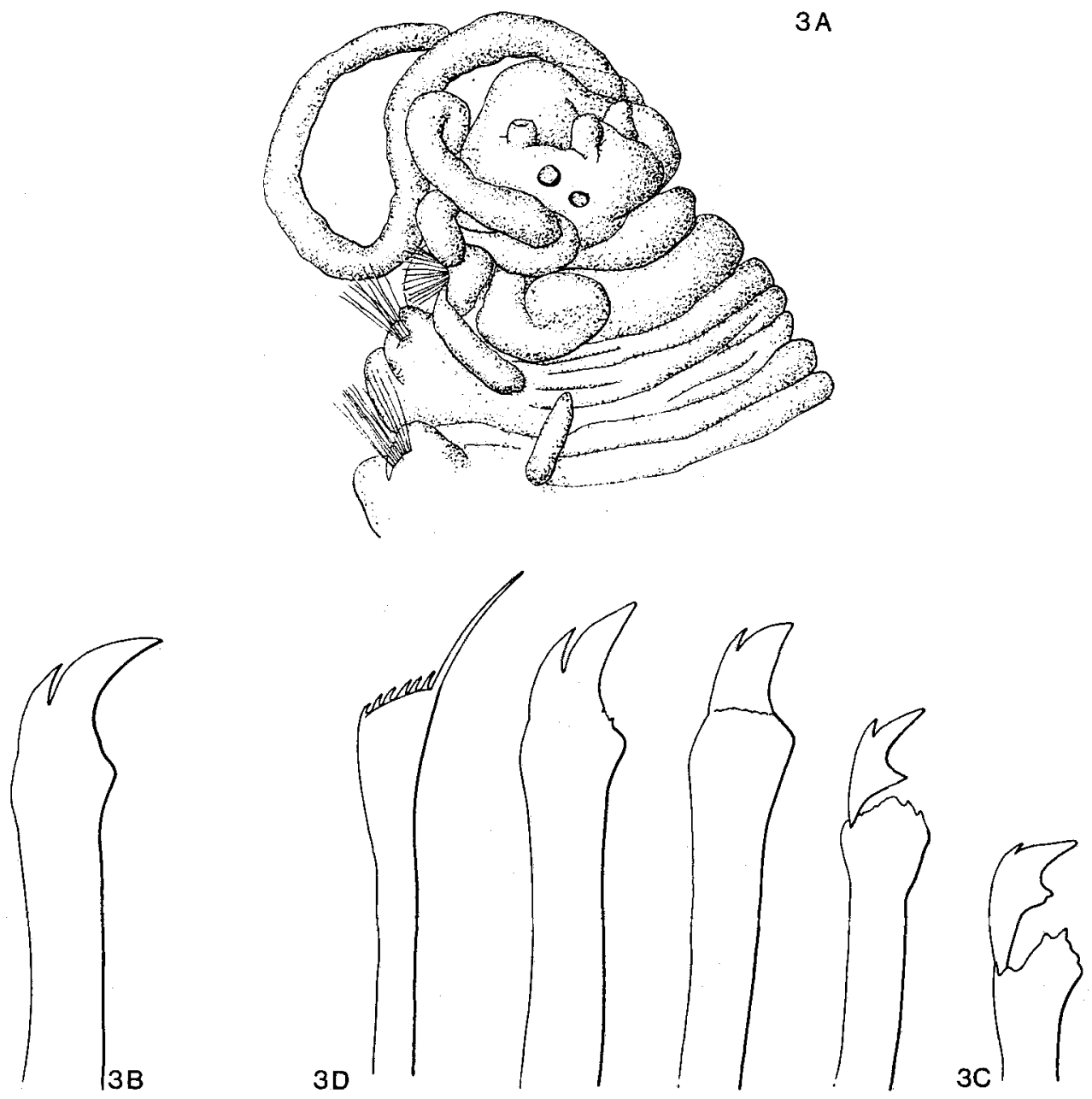

Abb. 3. Procerastea hydrozoicola n. sp. A: Vorderende (schräg dorsal) (laterale Antennen und 1. Dorsalcirrus fehlend, mediane Antenne lose neben der Ceratophore); B: einfache Borste (1. Parapod); C: untere Borste (vorderes Parapod); D: Borsten aus 11. Parapod

Paratypen von SMIB: 1. langgestrecktes Tier (nicht sicher, ob hinten einige Segmente fehlen) mit 13 Borstensegmenten $5,5 \mathrm{~mm}$ lang. Kräftig braun pigmentiert. Mediane Antenne lang, laterale Antennen abgebrochen. 1. Dorsalcirren lang, gewunden, etwas dicker als Tentakelcirren. Folgende Dorsalcirren wie beim Holotypus. Borsten zahlreich wie beim Holotypus. Einfache obere Borste auch im letzten vorhandenen Segment noch nicht entwickelt. Trepan nicht genau zu erkennen. Magen von der Mitte des 4. bis Ende des 5. Borstensegments, mit 21 Muskelzellreihen.

2. Sacconereis mit großen Augen, mit 30 Borstensegmenten $3,8 \mathrm{~mm}$ lang. Braunes Pigment besonders dorsal auf dem Vorderkörper. 1. Dorsalcirren lang und etwas dicker 
als übrige; Dorsalcirren an vorderen sieben und hinteren zehn Segmenten vorhanden, am mittleren Körper anscheinend abgebrochen. Kein Darm vorhanden. Zahl der Borsten nur 5-7, davon 1-2 untere zusammengesetzt. Einfache obere Borste ab 1. Parapod.

Fundort: SMIB 5, Stat. DW 103: Banc Aztèque auf der Norfolk-Rippe $\left(23^{\circ} 17,4^{\prime} \mathrm{S}-168^{\circ} 04,8^{\prime} \mathrm{O}\right): 315 \mathrm{~m}, 14$. 9. 1989: 2 Paratypen (ZMH: P-20 631).

T ax o n om is che Bemerkung: Procerastea cirrata Hartmann-Schröder und $P$. parasimpliseta sp. n. (s. u.) sind die einzigen Arten der Gattung, die ebenfalls Dorsalcirren an allen Segmenten besitzen. Die übrigen Arten haben Dorsalcirren nur am 1. Borstensegment entwickelt, abgesehen vom Sacconereis-Stadium von $P$. simpliseta HartmannSchröder, bei dem Dorsalcirren sowohl am 1. als auch vom 6. bis 15. Parapod vorhanden sind. Bei $P$. cirrata sind die Dorsalcirren im Gegensatz zu der hier neu beschriebenen Art unterschiedlich geformt: dick-spindelförmig die einen und zylindrisch die anderen. Außerdem sind bei $P$. cirrata die Borsten anders gestaltet: Der sekundäre Zahn ist sehr klein bis winzig, während hier der terminale kleiner ist. Weitere Diskussion siehe unten.

Procerastea parasimpliseta sp. $n$.

P. parasimpliseta sp. n. (Abb. 4). Holoty p u mit 20 Borstensegmenten (hinten unvollständig) 3,0 mm lang; größte Breite ohne Parapodien 0,31 mm. Farblos. Prostomium fast kreisförmig, Palpen von dorsal nicht sichtbar (Abb. 4A). Vier Augen im Trapez angeordnet, auf der Mitte des Prostomiums, die vorderen etwas größer. Neben den Augen einige kleine Pigmentkörnchen. Mediane Antenne abgebrochen, zwischen den vier Augen inserierend. Laterale Antennen am Vorderrand des Prostomiums, zylindrisch, gerunzelt, etwa eineinhalbmal so lang wie das Prostomium. Nuchalorgane am Hinterrand des Prostomiums nicht zu erkennen. 1. Segment nur lateral neben dem Prostomium sichtbar; dorsale Tentakelcirren etwas kürzer als die lateralen Antennen, ventrale nur halb so lang (Abb. 4 A). Parapodien sehr kurz, ventrolateral. 1. Dorsalcirren abgebrochen, wahrscheinlich etwas länger als übrige. Folgende Parapodien ebenfalls mit Dorsalcirren; diese jedoch kurz, oval (Abb. 4B). 8-14 Borsten pro Parapod, alle einfach, subdistal verbreitert und mit kleinen Zähnchen versehen; das verwachsene "Endglied" bifid, mit etwas größerem sekundären Zahn (Abb. $4 \mathrm{C}$ ). Einfache obere Borste distal verbreitert und mit etwa neun kleinen und einem längeren gebogenen endständigen Zahn oder Fortsatz (Abb. 4D), erst ab Körpermitte auftretend. Kein Darm vorhanden, möglicherweise jedoch unreife Gameten in der Leibeshöhle. Es handelt sich darum wohl um einen frühzeitig losgelösten Stolon.

Paraty p mit 27 Borstensegmenten 2,4 mm lang. Ebenfalls ohne Darm, anscheinend mit Gameten. Einfache obere Borste ab 1. Parapod vorhanden. Sonst kein Unterschied zum Holotypus.

L o cus ty pi cus : Musorstom 6, Stat. DW 472: Mont Musorstom auf der Rippe der Illes Loyauté $\left(21^{\circ} 60^{\prime} \mathrm{S}-167^{\circ} 54,70^{\prime} \mathrm{O}\right): 300 \mathrm{~m}, 22.2$. 1989: Holotypus (MNHN: UC 226) und 1 Paratyp (ZMH: P-20 632).

T a $\mathrm{x}$ on om is che B e m e r kung: Wie bereits oben erwähnt, gehört $P$. parasimpliseta sp. n. zusammen mit $P$. cirrata Hartmann-Schröder und $P$. hydrozoicola sp. n. zu den Arten der Gattung, die Dorsalcirren an allen Borstensegmenten entwickelt haben. P. parasimpliseta sp. n. unterscheidet sich von den beiden anderen Arten durch das Fehlen von zusammengesetzten Borsten, durch die Form der einfachen Borsten und die sehr kleinen Dorsalcirren. Auch P. pori Ben-Eliahu and P. simpliseta Hartmann-Schröder 
haben nur einfache Borsten; bei ihnen sind jedoch nur am 1. Borstensegment Dorsalcirren vorhanden - mindestens beim ungeschlechtlichen (atoken) Tier.

Sehr ähnlich ist Paraprocerastea crocantinae San Martin \& Alós. Diese Art hat lange Dorsalcirren am 1. und kleine ovale Dorsalcirren ab 2. Borstensegment. Bis auf die einfache, obere Borste, welche schmäler ist, sehen die Borsten genau so aus wie bei $P$. parasimpliseta sp. $\mathrm{n}$. Unterschiede: mit 3-5 ist die Zahl der Borsten deutlich geringer bei Paraprocerastea crocantinae; dort ist eine Nuchallamelle vorhanden, die bei $P$. parasimpliseta nicht zu erkennen ist, und das Gleiche gilt für Darm und Trepan, die bei der neuen Art fehlen, vermutlich weil es sich um einen Stolon handelt. Insgesamt scheinen beide Arten jedoch sehr nahe zueinander zu stehen.

San Martin \& Alós (1989) haben die Gattung Paraprocerastea für ihre neue Art $P$. crocantinae aufgestellt. Die Gattung soll sich von der Gattung Procerastea Langerhans in folgenden Merkmalen unterscheiden: neben langen 1. Dorsalcirren kleine Dorsalcirren an allen folgenden Segmenten; am Hinterrand des Prostomiums eine Nuchallamelle anstelle von Nuchalepauletten. Procerastea cirrata besitzt demnach Merkmale beider Gattungen: Dorsalcirren an allen Borstensegmenten wie Paraprocerastea und Nuchalepauletten wie Procerastea. Außerdem treten mehr als 1 Paar Dorsalcirren auch bei Stolonen von Arten auf, deren ungeschlechtliches Stadium Dorsalcirren nur am 1. Borstensegment entwickelt haben (wie z. B. bei $P$. simpliseta), und nach Allen (1921) haben regenerierte Segmente dieser Arten ebenfalls Dorsalcirren. Aus diesen Gründen

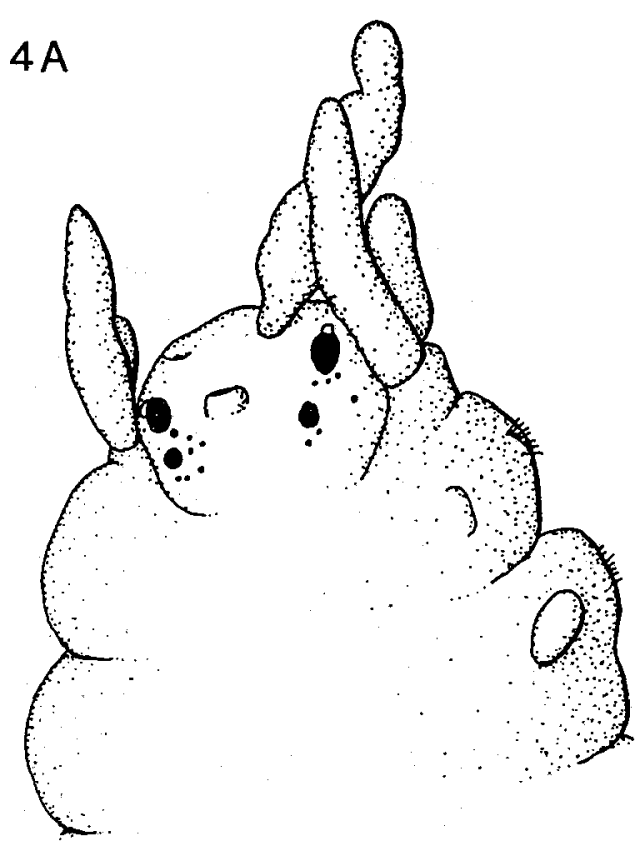

$4 B$

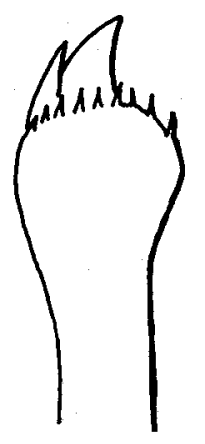

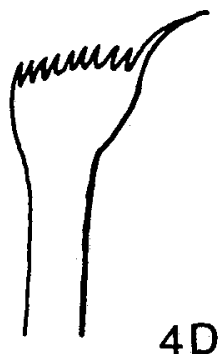

$4 \mathrm{D}$

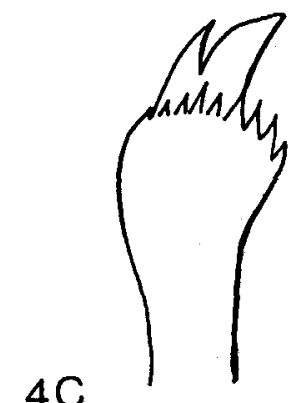

Abb. 4. Procerastea parasimpliseta n. sp. A: Vorderende von dorsal (2 Antennen und 1. Dorsalcirrus abgebrochen); B: Dorsalcirrus; C: Borsten; D: einfache obere Borste 
halte ich die Errichtung der Gattung Paraprocerastea nicht für gerechtfertigt und schlage hingegen eine erweiterte Diagnose der Gattung Procerastea vor (s. oben).

Die nahe verwandte Gattung Proceraea Ehlers besitzt ebenfalls Dorsalcirren an allen Borstensegmenten. Sie unterscheidet sich von der Gattung Procerastea jedoch durch das Fehlen von einfachen Borsten. Ihre Borsten ähneln denen der Autolytus-Arten: kurze Endglieder mit großem sekundären Zahn und kleinem bis winzigem terminalen Zahn.

Danksagung. Dr. H. Zibrowius danke ich für die Überlassung des Polychaetenmaterials und E. Quintero für die Übertragung der Bleistiftzeichnungen in Tintenstrichzeichnungen.

\section{LITERATUR}

Allen, E. J., 1921. Regeneration and reproduction of the syllid Procerastea. - Phil. Trans. R. Soc. London $211,131-177$.

Richer de Forges, B., 1990. Les campagnes d'exploration de la faune bathyale dans la zone économique de la Nouvelle-Calédonie. - Mém. Mus. natn. Hist. nat., Paris (Sér. A) 145, 9-54.

San Martin, G. \& Alós, S., 1989. Paraprocerastea crocantinae, a new genus and species (Polychaeta: Syllidae: Autolytinae) from the Spanish Mediterranean. - Proc. biol. Soc. Wash. 102, 872-877. 